\title{
REGULARIZATION METHOD FOR PARABOLIC EQUATION WITH VARIABLE OPERATOR
}

\author{
VALENTINA BURMISTROVA
}

Received 22 October 2004 and in revised form 28 June 2005

Consider the initial boundary value problem for the equation $u_{t}=-L(t) u, u(1)=w$ on an interval $[0,1]$ for $t>0$, where $w(x)$ is a given function in $L^{2}(\Omega)$ and $\Omega$ is a bounded domain in $\mathbb{R}^{n}$ with a smooth boundary $\partial \Omega$. $L$ is the unbounded, nonnegative operator in $L^{2}(\Omega)$ corresponding to a selfadjoint, elliptic boundary value problem in $\Omega$ with zero Dirichlet data on $\partial \Omega$. The coefficients of $L$ are assumed to be smooth and dependent of time. It is well known that this problem is ill-posed in the sense that the solution does not depend continuously on the data. We impose a bound on the solution at $t=0$ and at the same time allow for some imprecision in the data. Thus we are led to the constrained problem. There is built an approximation solution, found error estimate for the applied method, given preliminary error estimates for the approximate method.

\section{Introduction}

Consider the problem of solving a parabolic partial differential equation with variable operator backwards in time. For convenience we write the equation in the following abstract form

$$
\begin{gathered}
u_{t}=-L(t) u, \quad 0 \leq t \leq 1, \\
u(1)=w .
\end{gathered}
$$

Here $w(x)$ is a given function in $L^{2}(\Omega)$, and $\Omega$ is a bounded domain in $\mathbb{R}^{n}$ with a smooth boundary $\partial \Omega$. $L$ is the unbounded, nonnegative operator in $L^{2}(\Omega)$ corresponding to a selfadjoint, elliptic boundary value problem in $\Omega$ with zero Dirichlet data on $\partial \Omega$. The coefficients of $L$ are assumed to be smooth and dependent of time.

The system (1.1) is ill-posed because the solution does not depend continuously on the data. We impose a bound on the solution at $t=0$ and at the same time allow for some imprecision in the data. Now we are led to the constrained problem. 
Get any solution of

$$
\begin{gathered}
u_{t}=-L(t) u, \quad 0 \leq t \leq 1, \\
\|u(1)-w\| \leq \delta, \\
\|u(0)\| \leq M
\end{gathered}
$$

where the norm is the $L^{2}(\Omega)$-norm, and $\delta$ and $M$ are given positive constants, $\delta \ll M$. Using logarithmic convexity (see [1], [7, page 11]), we have that any two solutions of (1.2), $u_{1}$ and $u_{2}$, satisfy

$$
\left\|u_{1}(t)-u_{2}(t)\right\| \leq 2 \delta^{t} M^{1-t}
$$

Write down system

$$
\begin{gathered}
u_{t}=-L(t) u, \quad 0 \leq t \leq 1, \\
w=u(0) e^{-\int_{0}^{1} L(\tau) d \tau}+\psi, \\
\|\psi\| \leq \delta \\
\|u(0)\| \leq M \leq \delta
\end{gathered}
$$

Thus for $0<t \leq 1$ we have continuous dependence on the data.

It is difficult to solve (1.2), because solutions are not unique. There are some methods for approximating solutions of (1.2), which are optimal in the sense that Hölder type error estimates (1.3) can be obtained for them.

We consider a method related to the regularization method of Tikhonov [5] and Phillips. This method for parabolic equation with operator $L$ independent of time is learned in [4]. Now we consider more generalized case: parabolic equation with variable coefficients.

An approximate solution of (1.2) is given by

$$
v(t)=\frac{e^{-\int_{0}^{t} L(\tau) d \tau}}{e^{-\int_{0}^{1} L(\tau) d \tau}+\mu(t)} w, \quad \mu(t)=(\delta / M)(1-t) / t
$$

Let $u$ be any solution of (1.2). Then, for $0 \leq t \leq 1$,

$$
\begin{gathered}
\|u(t)-v(t)\| \leq \delta^{t} M^{1-t}, \\
\|u-v\|=\left\|u_{0} e^{-\int_{0}^{t} L(\tau) d \tau}-\frac{e^{-\int_{0}^{t} L(\tau) d \tau}}{e^{-\int_{0}^{1} L(\tau) d \tau}+\mu(t)}\left(u_{0} e^{-\int_{0}^{1} L(\tau) d \tau}\right)+\psi\right\| \\
=\left\|e^{-\int_{0}^{t} L(\tau) d \tau}-\frac{e^{-\int_{0}^{t} L(\tau) d \tau-\int_{0}^{1} L(\tau) d \tau}}{e^{-\int_{0}^{1} L(\tau) d \tau}+\mu(t)}\right\|\left\|u_{0}\right\|+\left\|\frac{e^{-\int_{0}^{t} L(\tau) d \tau}}{e^{-\int_{0}^{1} L(\tau) d \tau}+\mu(t)}\right\|\|\psi\| .
\end{gathered}
$$


We now raise the following question. Can we discretize (1.5) in such a way that for the discrete approximation $v_{a}$ we get an error estimate of type (1.6)

$$
\|u(t)-v(t)\| \leq C \delta^{t} M^{1-t}
$$

for some constant $C$ ?

The answer to this question will have significance for the possibilities of solving numerically problems in two (or more) space dimensions, with nonrectangular geometry or nonconstant coefficients, since for such problems we must discretize in time and space.

In this paper, we give a partial answer to the above question. We consider approximating the exponential function in (1.5) in a way which corresponds to a time discretization. In Section 3, we show that if $\exp (-\lambda)$ is approximated well enough for $0 \leq \lambda \leq \log (M / \delta)$, we can get error estimates of the form (1.7) with $C=2$.

\section{The regularization method for parabolic equation with variable coefficients}

We show that the estimate (1.6) holds for the regularization method (1.5). The proof is quite simple and we use the same method in connection with discretization of (1.5). We also show that the same error estimate is valid if we use (1.5) in a step-by-step manner.

We assume that $\delta$ and $M$ have been chosen so that there exist solutions of (1.2).

Theorem 2.1. Let $u(t)$ denote an arbitrary solution of (1.2), and for $0 \leq t \leq 1$ let $v(t)$ be defined by (1.5). Then

$$
\|u(t)-v(t)\| \leq \delta^{t} M^{1-t}
$$

Proof. The assumption about the existence of solutions of (1.2) is equivalent to there being functions $u_{0}$ and $\psi$ such that

$$
\left\|u_{0}\right\| \leq M, \quad\|\psi\| \leq \delta, \quad w=\exp (-L) u_{0}+\psi
$$

Putting $u(t)=u_{0} e^{-\int_{0}^{t} L(\tau) d \tau}$ we get

$$
\begin{aligned}
\|u-v\| & =\left\|u_{0} e^{-\int_{0}^{t} L(\tau) d \tau}-\frac{e^{-\int_{0}^{t} L(\tau) d \tau}}{e^{-\int_{0}^{1} L(\tau) d \tau}+\mu(t)}\left(u_{0} e^{-\int_{0}^{1} L(\tau) d \tau}\right)+\psi\right\| \\
& =\left\|e^{-\int_{0}^{t} L(\tau) d \tau}-\frac{e^{-\int_{0}^{t} L(\tau) d \tau-\int_{0}^{1} L(\tau) d \tau}}{e^{-\int_{0}^{1} L(\tau) d \tau}+\mu(t)}\right\|\left\|u_{0}\right\|+\left\|\frac{e^{-\int_{0}^{t} L(\tau) d \tau}}{e^{-\int_{0}^{1} L(\tau) d \tau}+\mu(t)}\right\|\|\psi\|,
\end{aligned}
$$

where the operator norm is defined such way $\|A\|=\sup \{\|A u\|:\|u\|=1\}$. We now use (2.2) and the fact that $L$ is selfadjoint and nonnegative to get

$$
\|u(t)-v(t)\| \leq \sup _{\lambda \geq 0} A(\lambda) M+\sup _{\lambda \geq 0} B(\lambda) \delta,
$$


where

$$
\begin{aligned}
& A(\lambda)=\left|e^{-\int_{0}^{t} L(\tau) d \tau}-\frac{e^{-\int_{0}^{t} L(\tau) d \tau-\int_{0}^{1} L(\tau) d \tau}}{e^{-\int_{0}^{1} L(\tau) d \tau}+\mu(t)}\right|, \\
& B(\lambda)=\frac{e^{-\int_{0}^{t} L(\tau) d \tau}}{e^{-\int_{0}^{1} L(\tau) d \tau}+\mu(t)}
\end{aligned}
$$

We have $A=\mu(t) e^{-\int_{0}^{t} L(\tau) d \tau} /\left(e^{-\int_{0}^{1} L(\tau) d \tau}+\mu(t)\right)=\mu(t) B$.

Let $p=e^{-\int_{0}^{1} L(\tau) d \tau} /\left(e^{-\int_{0}^{1} L(\tau) d \tau}+\mu(t)\right), 1-p=\mu(t) /\left(e^{-\int_{0}^{1} L(\tau) d \tau}+\mu(t)\right)$. We use the fact from [4]. We have for $0 \leq p, t \leq 1$ the inequality

$$
p^{t}(1-p)^{1-t} \leq t^{t}(1-t)^{1-t}
$$

is valid, we obtain

$$
\begin{aligned}
B(\lambda) & =\frac{e^{-\int_{0}^{t} L(\tau) d \tau}}{e^{-\int_{0}^{1} L(\tau) d \tau}+\mu(t)} \leq \frac{e^{-t \int_{0}^{t} \chi L(\tau) d \tau}}{e^{-\int_{0}^{1} L(\tau) d \tau}+\mu(t)} \\
& =\left(\frac{e^{-\int_{0}^{t} \chi L(\tau) d \tau}}{e^{-\int_{0}^{1} L(\tau) d \tau}+\mu(t)}\right)^{t}\left(\frac{\mu(t)}{e^{-\int_{0}^{1} L(\tau) d \tau}+\mu(t)}\right)^{1-t} \mu^{t-1}(t) \\
& \leq t^{t}(1-t)^{1-t}\left(\frac{\delta}{M}\right)^{t-1}\left(\frac{1-t}{t}\right)^{t-1}=t\left(\frac{M}{\delta}\right)^{1-t}, \\
A & \leq \frac{\delta}{M} \frac{1-t}{t} t\left(\frac{M}{\delta}\right)^{1-t}=\left(\frac{\delta}{M}\right)^{t}(1-t)
\end{aligned}
$$

(look at the definition (1.5) of $\mu(t)$ ). Therefore we can estimate (2.4)

$$
\|u(t)-v(t)\| \leq\left(\frac{\delta}{M}\right)^{t}(1-t) M+t\left(\frac{M}{\delta}\right)^{1-t} \delta=\delta^{t} M^{1-t} .
$$

The numerical of a forward parabolic problem is usually computed by a marching procedure, that is, a procedure which is recursive in time. We show that the method (1.5) for the backward problem can be generalized to a recursive formula in such a way that the procedure remains optimal in the above sense. Make a (possibly nonuniform) partitioning of the interval $[0,1]$

$$
0<t_{1}<t_{2}<\cdots<t_{s}<1
$$

and let the recursion be

$$
v_{s}=v\left(t_{s}\right), \quad v_{i-1}=\frac{e^{-\int_{0}^{t_{i}-1} L(\tau) d \tau}}{e^{-\int_{0}^{t_{i}} L(\tau) d \tau}+\mu_{i}} v_{i}, \quad i=s, s-1, \ldots, 2,
$$


where $v\left(t_{s}\right)$ is given by $(1.5)$ and

$$
\mu_{i}=\left(\delta_{i} / M\right)\left(t_{i}-t_{i-1}\right) / t_{i-1}, \quad \delta_{i}=\delta^{t_{i}} M^{1-t_{i}} .
$$

Corollary 2.2. Let $u(t)$ denote an arbitrary solution of (1.2), and let $\left(v_{i}\right)_{i=1}^{s}$ be defined by (2.10). Then

$$
\left\|u\left(t_{i}\right)-v_{i}\right\| \leq \delta^{t_{i}} M^{1-t_{i}}
$$

Proof. The result is obviously true for $i=s$. Then assume that it is true for $i=k$, and consider

$$
u_{t}=-L(t) u \quad \text { for } 0<t \leq t_{k}, \quad\left\|u\left(t_{k}\right)-v_{k}\right\| \leq \delta_{k}, \quad\|u(0)\| \leq M .
$$

The recursion formula (2.10) is a straightforward generalization of (1.5) to the interval $\left[0, t_{k}\right]$, and, putting $\tau_{k}=t_{k-1} / t_{k}$, we obtain

$$
\left\|u\left(t_{k-1} v_{k-1}\right)\right\| \leq \delta^{\tau_{k}} M^{1-\tau_{k}}=\delta^{t_{k-1}} M^{1-t_{k-1}}=\delta_{k-1} .
$$

\section{Preliminary error estimation}

We get now error estimates for

$$
v=\frac{e^{-\int_{0}^{t} L(\tau) d \tau}}{e^{-\int_{0}^{1} L(\tau) d \tau}+\mu(t)} \leq \frac{e^{-t \int_{0}^{1} L(\tau) d \tau}}{e^{-\int_{0}^{1} L(\tau) d \tau}+\mu(t)} .
$$

Let

$$
v_{a}(t)=\frac{g^{t}}{g+\mu(t)} w, \quad g \approx e^{-\int_{0}^{1} L(\tau) d \tau}
$$

which is (1.5) with the exponential function replaced by an approximation $f$, such that $f(\lambda) \approx e^{-\lambda}$. In the next section $f(\lambda)$ will depend on $N$, where $k=1 / N$ is a step length parameter, but here this dependence is suppressed. There we will be dealing explicitly with the class of approximations defined by $\exp -\lambda \approx(Q(\lambda / N) / P(\lambda / N))^{N}($ see $[1$, p. 54] $)$, but in this section it will be sufficient to distinguish between two subclasses characterized by the following inequalities:

$$
\begin{gathered}
e^{-\int_{0}^{t} \lambda(\tau) d \tau} \leq g(\lambda) \leq 1, \quad \lambda \geq 0, \\
0<g(\lambda) \leq e^{-\int_{0}^{t} \lambda(\tau) d \tau}, \quad 0 \leq e^{\int_{0}^{t} \lambda(\tau) d \tau} \leq \ln (M / \delta), \\
0 \leq g(\lambda) \leq 1, \quad e^{\int_{0}^{t} \lambda(\tau) d \tau} \geq \ln (M / \delta) .
\end{gathered}
$$

First we give an error estimate for approximations satisfying (3.3). 
Theorem 3.1. Let $u(t)$ denote an arbitrary solution of (1.2), let $v_{a}(t)$ be defined by (3.2), and assume that $f$ satisfies (3.3). If

$$
\lambda+\ln g(\lambda) \leq(\delta / M) \frac{1}{t} e^{\lambda t} \quad \text { for } 0 \leq \lambda \leq \ln (M / \delta)
$$

then

$$
\left\|u(t)-v_{a}(t)\right\| \leq(t+\max (1,2(1-t))) \delta^{t} M^{1-t} .
$$

Proof. As in Theorem 2.1 we have

$$
\left\|u(t)-v_{a}(t)\right\| \leq \sup _{\lambda \geq 0} A(\lambda) M+\sup _{\lambda \geq 0} B(\lambda) \delta
$$

Look at it in details

$$
\begin{aligned}
\left\|u(t)-v_{a}(t)\right\| & =\left\|u_{0} e^{-\int_{0}^{t} L(\tau) d \tau}-\frac{g^{t}}{g+\mu(t)}\left(e^{-\int_{0}^{1} L(\tau) d \tau}+\psi\right)\right\| \\
& \leq\left\|e^{-\int_{0}^{t} L(\tau) d \tau}-\frac{g^{t}}{g+\mu(t)} e^{-\int_{0}^{1} L(\tau) d \tau}\right\|\left\|u_{0}\right\|+\left\|\frac{g^{t}}{g+\mu(t)}\right\|\|\psi\| .
\end{aligned}
$$

Here

$$
\begin{aligned}
& A=\left\|e^{-\int_{0}^{t} \lambda(\tau) d \tau}-\frac{g^{t}}{g+\mu(t)} e^{-\int_{0}^{1} \lambda(\tau) d \tau}\right\|, \\
& B=\frac{g^{t}}{g+\mu(t)} .
\end{aligned}
$$

By (2.6) we have

$$
B \leq t\left(\frac{M}{\delta}\right)^{1-t}
$$

We have then $A=\left|A_{1}-A_{2}\right|, A_{1}=e^{-\int_{0}^{t} \lambda(\tau) d \tau}, A_{2}=\left(g^{t} /(g+\mu(t))\right) e^{-\int_{0}^{1} \lambda(\tau) d \tau}$. Let $\int_{0}^{1} \lambda(\tau) d \tau \geq k$, then, for $A_{1} \geq A_{2}$ we have $A \leq e^{-\int_{0}^{t} \lambda(\tau) d \tau} \leq e^{-t \int_{0}^{1} \lambda(\tau) d \tau} \leq e^{-k t}$; and for $A_{1} \leq$ $A_{2}$ we have $A \leq\left(g^{t} /(g+\mu(t))\right) e^{-\int_{0}^{1} \lambda(\tau) d \tau} \leq t(M / \delta)^{1-t} e^{-k}=t e^{k(1-t)} e^{-k}=t e^{-k t}$, therefore

$$
A \leq e^{-k t}
$$

Consider now case $\int_{0}^{1} \lambda(\tau) d \tau \leq k$. 
Let at first $A_{1} \geq A_{2}$, then $A=A_{1}-A_{2}$. We have then

$$
\begin{aligned}
A(g+\mu(t)) & =\mu(t) e^{-\int_{0}^{t} \lambda(\tau) d \tau}+g e^{-\int_{0}^{t} \lambda(\tau) d \tau}-g^{t} e^{-\int_{0}^{1} \lambda(\tau) d \tau} \\
& \leq \mu(t) e^{-t \int_{0}^{1} \lambda(\tau) d \tau}+g e^{-t \int_{0}^{1} \lambda(\tau) d \tau}-g^{t} e^{-\int_{0}^{1} \lambda(\tau) d \tau} \\
& =\mu(t) e^{-t \int_{0}^{1} \lambda(\tau) d \tau}+g e^{-t \int_{0}^{1} \lambda(\tau) d \tau}\left(g^{1-t}-e^{-(1-t) \int_{0}^{1} \lambda(\tau) d \tau}\right) \\
& \leq \mu(t) e^{-t \int_{0}^{1} \lambda(\tau) d \tau}+g e^{-t \int_{0}^{1} \lambda(\tau) d \tau}\left(\ln g+\int_{0}^{1} \lambda(\tau) d \tau\right)(1-t) \\
& \leq \mu(t) e^{-t \int_{0}^{1} \lambda(\tau) d \tau}+g e^{-t \int_{0}^{1} \lambda(\tau) d \tau} \frac{\delta}{M} \frac{1}{t} e^{-t \int_{0}^{1} \lambda(\tau) d \tau}(1-t) \\
& =\mu(t) e^{-t \int_{0}^{1} \lambda(\tau) d \tau}+g^{t} \mu(t) 0=\mu(t)\left(e^{-t \int_{0}^{1} \lambda(\tau) d \tau}+g^{t}\right) \leq 2 \mu(t) g^{t} \\
A & \leq 2 \mu(t) \frac{g^{t}}{g+\mu(t)} \leq 2 \frac{\delta}{M} \frac{1-t}{t} t\left(\frac{M}{\delta}\right)^{1-t}=2\left(\frac{\delta}{M}\right)^{t}(1-t)=2(1-t) e^{-k t}
\end{aligned}
$$

Let now $A_{1} \leq A_{2}$, then $A=A_{2}-A_{1}$. We have then

$$
\begin{aligned}
A(g+\mu(t)) & =g^{t} e^{-\int_{0}^{1} \lambda(\tau) d \tau}-g e^{-\int_{0}^{t} \lambda(\tau) d \tau} \mu(t) e^{-\int_{0}^{t} \lambda(\tau) d \tau} \\
& =g^{t} e^{-\int_{0}^{t} \lambda(\tau) d \tau}\left(e^{-\int_{t}^{1} \lambda(\tau) d \tau}-g^{1-t}\right)-\mu(t) e^{-\int_{0}^{t} \lambda(\tau) d \tau} \\
& =g^{t} e^{-\int_{0}^{t} \lambda(\tau) d \tau}\left(e^{-\lambda(\xi)(1-\tau)}-g^{1-t}\right)-\mu(t) e^{-\int_{0}^{t} \lambda(\tau) d \tau} \\
& \leq g^{t} e^{-\int_{0}^{t} \lambda(\tau) d \tau}(-\lambda)(-\lambda(\xi)-\ln g)(1-t)-\mu(t) e^{-\int_{0}^{t} \lambda(\tau) d \tau}
\end{aligned}
$$

Here if $-\lambda(\xi)-\ln g<0$, then $A<0$ and $A_{1} \geq A_{2}$, that is, we have earlier observed case.

$$
\begin{gathered}
\text { Let }-\lambda(\xi)-\ln g \leq(\delta / M)(1 / t) e^{\int_{0}^{t} \lambda(\tau) d \tau}, \int_{0}^{1} \lambda(\tau) d \tau \leq k=\ln (M / \delta), t \leq \xi \leq 1 \text {. Then } \\
\qquad \begin{array}{c}
A(g+\mu(t))=g^{t} e^{-\int_{0}^{1} \lambda(\tau) d \tau} \leq \frac{\delta}{M} \frac{1}{t} e^{\int_{0}^{t} \lambda(\tau) d \tau}(1-t)-\mu(t) e^{-\int_{0}^{t} \lambda(\tau) d \tau} \leq g^{t} \mu(t), \\
A \leq \frac{g^{t}}{g+\mu(t)} \mu(t)=(1-t) e^{-k t} .
\end{array}
\end{gathered}
$$

Thus,

$$
\begin{gathered}
A \leq 2(1-t)\left(\frac{\delta}{M}\right)^{t}, \\
\left\|u(t)-v_{a}(t)\right\| \leq 2(1-t)(\delta M)^{t} M+t(M \delta)^{1-t} \delta=(t+\max (1,2(1-t))) \delta^{t} M^{1-t} .
\end{gathered}
$$

We next give the corresponding theorem for approximations satisfying (3.4). 
Theorem 3.2. Let $u(t)$ denote an arbitrary solution of (1.2), let $v_{a}(t)$ be defined by (3.2), and assume that $f$ satisfies (3.4). If

$$
-\int_{0}^{1} \lambda(\tau) d \tau-\ln g(\lambda) \leq \frac{\delta}{M} \frac{\ln 2}{t} e^{\int_{0}^{1} \lambda(\tau) d \tau} \quad \text { for } 0 \leq \lambda \leq \ln \frac{M}{\delta},
$$

then

$$
\left\|u(t)-v_{a}(t)\right\| \leq(t+\max (1,2(1-t))) \delta^{t} M^{1-t} .
$$

Remark 3.3. The assumption (3.4) implies that $-\lambda-\log f(\lambda)$ is nonnegative.

Proof. As in proof of Theorem 3.1 we get

$$
\left\|u(t)-v_{a}(t)\right\| \leq \sup _{\lambda \geq 0} A(\lambda) M+\sup _{\lambda \geq 0} B(\lambda) \delta
$$

where $A(\lambda)$ and $B(\lambda)$ are the same.

$$
B \leq t\left(\frac{M}{\delta}\right)^{1-t}
$$

If $\int_{0}^{1} \lambda(\tau) d \tau \geq k$, then $A \leq e^{-k t}=(\delta / M)^{t}$.

If $\int_{0}^{1} \lambda(\tau) d \tau \geq k$, then let at first $A=A_{1}-A_{2}$, and so we have

$$
\begin{aligned}
A(g+\mu(t)) & =\mu(t) e^{-\int_{0}^{t} \lambda(\tau) d \tau}+g e^{-\int_{0}^{t} \lambda(\tau) d \tau}-g^{t} e^{-\int_{0}^{1} \lambda(\tau) d \tau} \\
& \leq \mu(t) e^{-t \int_{0}^{1} \lambda(\tau) d \tau}+g^{t} e^{-t \int_{0}^{1} \lambda(\tau) d \tau}\left(g^{1-t}-e^{-(1-t) \int_{0}^{1} \lambda(\tau) d \tau}\right) \\
& \leq \mu(t) e^{-t \int_{0}^{t} \lambda(\tau) d \tau} .
\end{aligned}
$$

As we have

$$
0 \leq \int_{0}^{1} \lambda(\tau) d \tau \leq \ln \frac{M}{\delta}=k
$$

so $e^{\int_{0}^{1} \lambda(\tau) d \tau}(\delta / M) \leq 1$, and accounting condition of theorem

$$
-\int_{0}^{1} \lambda(\tau) d \tau-\ln g(\lambda) \leq \frac{\delta}{M} \frac{\ln 2}{t} e^{\int_{0}^{1} \lambda(\tau) d \tau}
$$

for $\int_{0}^{1} \lambda(\tau) d \tau \leq k$ we have $-\int_{0}^{1} \lambda(\tau) d \tau-\ln g(\lambda) \leq \ln 2 / t$, therefore

$$
-t \int_{0}^{1} \lambda(\tau) d \tau-t \ln g(\lambda) \leq \ln 2, \quad-t \int_{0}^{1} \lambda(\tau) d \tau \leq \ln 2+t \ln g, \quad e^{-t \int_{0}^{1} \lambda(\tau) d \tau} \leq 2 g^{t},
$$


and, hence,

$$
A(g+\mu(t)) \leq \mu(t) \cdot 2 g^{t}, \quad A \leq 2 \mu(t) \frac{g^{t}}{g+\mu(t)} \leq 2(1-t)\left(\frac{\delta}{m}\right)^{t} .
$$

Let now $A=A_{2}-A_{1}$. Then

$$
\begin{aligned}
A(g+\mu(t))= & g^{t} e^{-\int_{0}^{1} \lambda(\tau) d \tau}-g e^{-\int_{0}^{t} \lambda(\tau) d \tau}-\mu(t) e^{-\int_{0}^{t} \lambda(\tau) d \tau} \\
= & g^{t} e^{-\int_{0}^{1} \lambda(\tau) d \tau}\left(e^{-\int_{t}^{1} \lambda(\tau) d \tau}-g^{1-t}\right) \\
& -\mu(t) e^{-\int_{0}^{t} \lambda(\tau) d \tau} g^{t} e^{-\int_{0}^{1} \lambda(\tau) d \tau}\left(e^{-\int_{t}^{1} \lambda(\xi) d \tau}-g^{1-t}\right)-\mu(t) e^{-\int_{0}^{t} \lambda(\tau) d \tau} \\
\leq & g^{t} e^{-\int_{0}^{1} \lambda(\tau) d \tau}(-\lambda(\xi)-\ln g)-\mu(t) e^{-\int_{0}^{t} \lambda(\tau) d \tau} \\
= & g^{t} e^{-\int_{0}^{1} \lambda(\tau) d \tau} \frac{\delta}{M} \frac{1}{t} e^{\int_{0}^{1} \lambda(\tau) d \tau}(1-t)-\mu(t) e^{-\int_{0}^{t} \lambda(\tau) d \tau} \\
= & \mu(t)\left(g^{t}-e^{-\int_{0}^{t} \lambda(\tau) d \tau}\right) \leq \mu t g^{t},
\end{aligned}
$$

then $A \leq \mu(t)\left(g^{t} /(g+\mu(t))\right) \leq(1-t)(\delta / M)^{t}$.

So, we have $A \leq 2(1-t)(\delta / M)^{t}$ and

$$
\left\|u-v_{a}\right\| \leq 2(1-t)\left(\frac{\delta}{M}\right)^{t} M+t\left(\frac{M}{\delta}\right)^{1-t} \delta=(t+\max 1,2(1-t)) \delta^{t} M^{1-t} .
$$

Thus we obtain the same error estimate as for case with operator independent of time [4]. It means that the method can be applied for more wide field of problems.

\section{Acknowledgments}

The results of this paper were obtained during my Fulbright research at Iowa State University. The author takes pleasure in thanking Professor Howard Levine for his guidance and support.

\section{References}

[1] S. Agmon and L. Nirenberg, Properties of solutions of ordinary differential equations in Banach space, Comm. Pure Appl. Math. 16 (1963), 121-239.

[2] B. L. Buzbee, Application of fast Poisson solvers to A-stable marching procedures for parabolic problems, SIAM J. Numer. Anal. 14 (1977), no. 2, 205-217.

[3] B. L. Buzbee and A. Carasso, On the numerical computation of parabolic problems for preceding times, Math. Comp. 27 (1973), 237-266.

[4] L. Eldén, Time discretization in the backward solution of parabolic equations. I, Math. Comp. 39 (1982), no. 159, 53-68.

[5] - Time discretization in the backward solution of parabolic equations. II, Math. Comp. 39 (1982), no. 159, 69-84.

[6] A. Friedman, Partial Differential Equations, Holt, Rinehart, and Winston, New York, 1969.

[7] L. E. Payne, Improperly Posed Problems in Partial Differential Equations, Regional Conference Series in Applied Mathematics, no. 22, SIAM, Philadelphia, 1975. 
[8] V. N. Strakhov, Solution of incorrectly-posed linear problems in Hilbert space, Differ. Equ. 6 (1970), 1136-1140 (Russian).

[9] A. N. Tikhonov and V. Ya. Arsenin, Methods of Decision of Ill-Posed Problems, Nauka, Moscow, 1986.

Valentina Burmistrova: International Research and Exchanges Board (IREX), 48 A Gerogoly Street, 744000 Ashgabat, Turkmenistan

E-mail addresses: valeb@mail.ru; valeb@iatp.edu.tm 


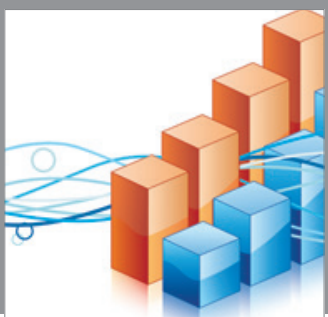

Advances in

Operations Research

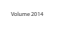

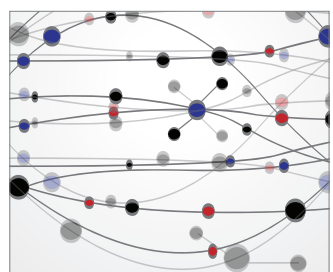

\section{The Scientific} World Journal
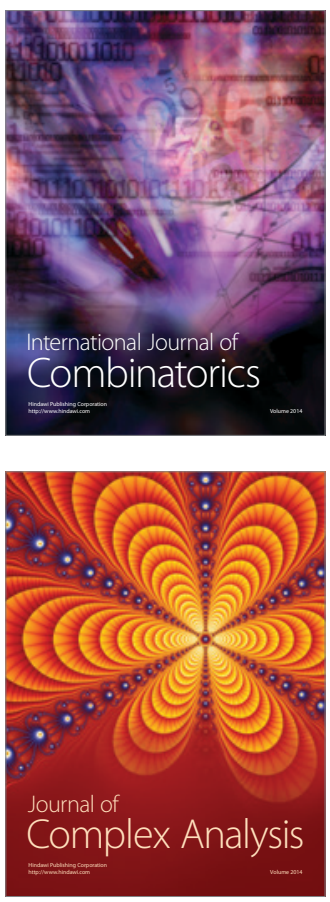

International Journal of

Mathematics and

Mathematical

Sciences
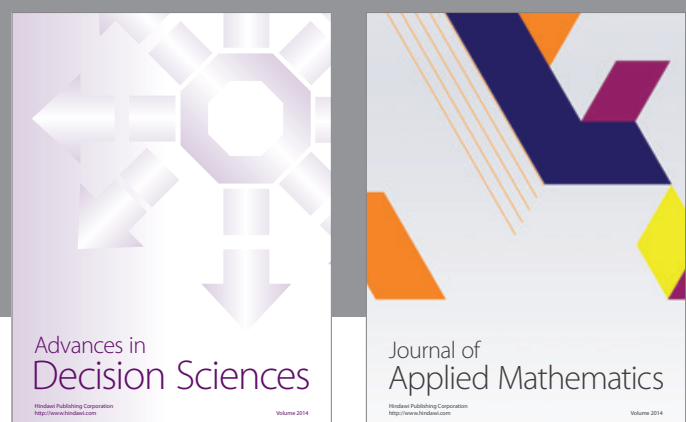

Journal of

Applied Mathematics
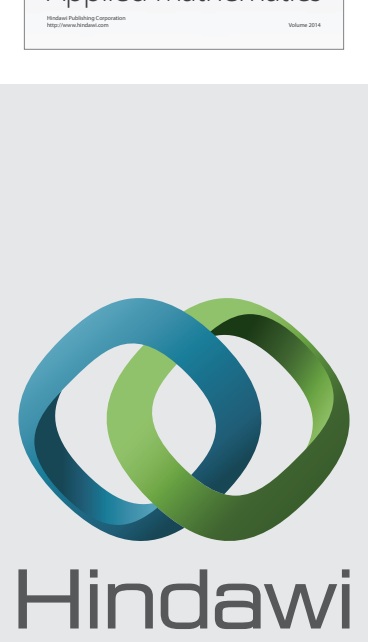

Submit your manuscripts at http://www.hindawi.com
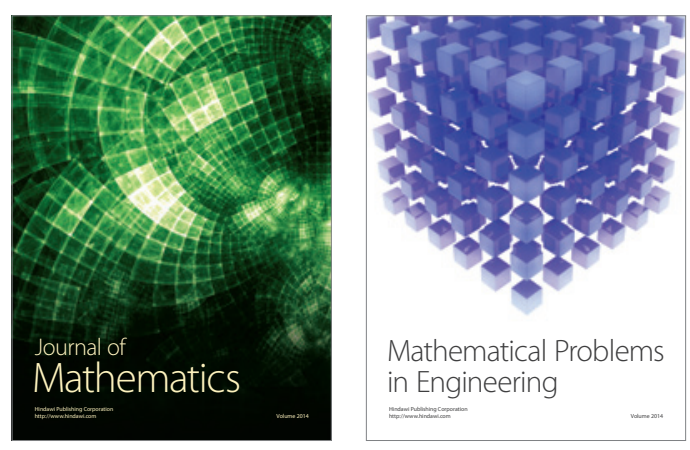

Mathematical Problems in Engineering
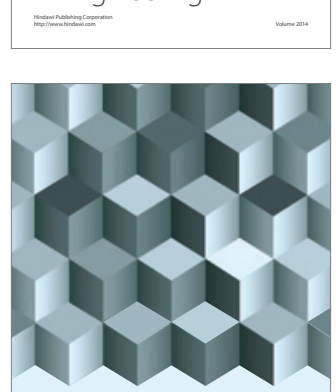

Journal of

Function Spaces
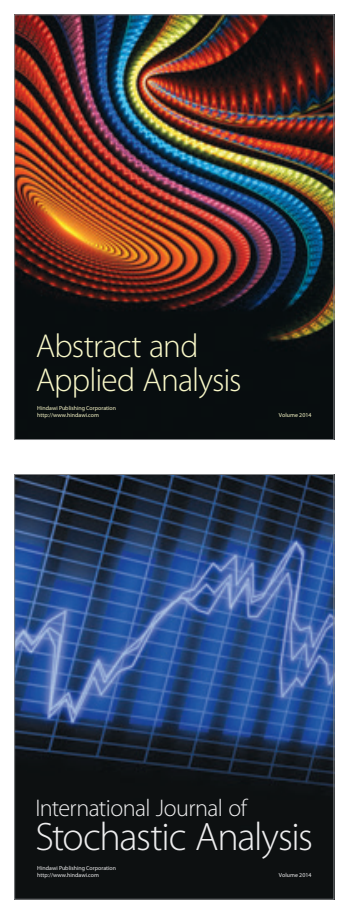

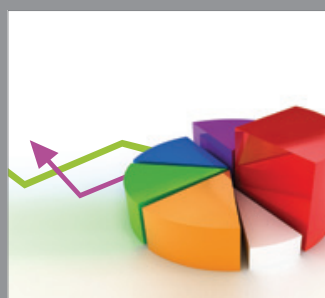

ournal of

Probability and Statistics

Promensencen
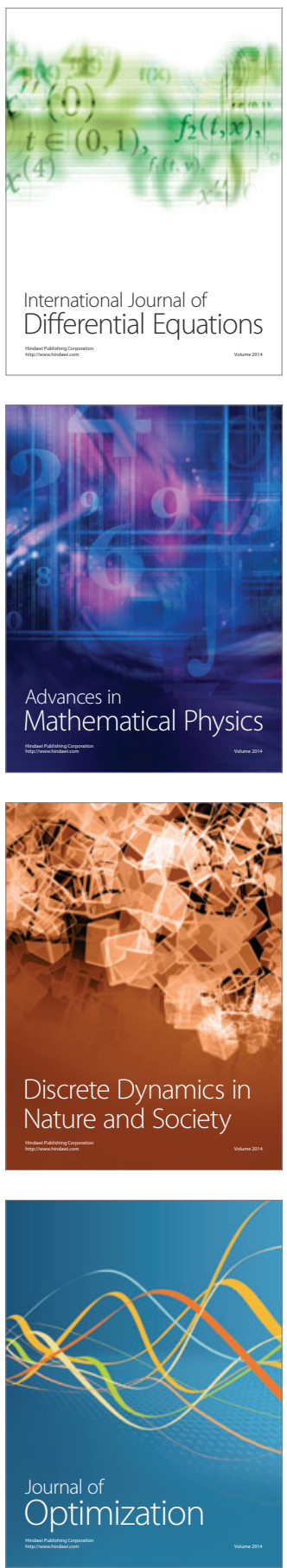